\title{
Suitable carriers for encapsulation and distribution of endostar: comparison of endostar-loaded particulate carriers
}

This article was published in the following Dove Press journal:

International Journal of Nanomedicine

21 July 20II

Number of times this article has been viewed

\section{Weijie Chen}

Sanyuan Hu

Department of General Surgery, Qilu Hospital of Shandong University, Jinan, Shandong Province,

People's Republic of China
Correspondence: Sanyuan Hu Department of General Surgery, Qilu Hospital of Shandong University, 107 Wenhua Xi Road, Jinan 2500I2, Shandong Province, People's Republic of China

Tel +8653186920598

$\mathrm{Fax}+8653182169293$

Email cwj022303019@I63.com
Background: Particulate carriers are necessary to control the release of endostar and prolong its circulation in vivo. The purpose of this study was to identify a suitable carrier for the capsulation and delivery of endostar.

Methods: We prepared a series of poly (DL-lactide-co-glycolide) (PLGA) and poly (ethylene glycol) (PEG)-modified PLGA (PEG-PLGA) particulate carriers, and then characterized them according to their ability to prolong the circulation of endostar, their physicochemical properties, endostar-loading content, and in vitro and in vivo particulate carrier release profiles.

Results: All the particulate carriers had spherical core shell structures. The PEG-PLGA material and nanosize range appeared to enable the carriers to encapsulate more endostar, release endostar faster in vitro, and accumulate more endostar in vivo. The drug loading capacity of PEG-PLGA and PLGA nanoparticles was $8.03 \% \pm 3.41 \%$ and $3.27 \% \pm 5.26 \%$, respectively, and for PEGPLGA and PLGA microspheres was $15.32 \% \pm 5.61 \%$ and $9.21 \% \pm 4.73 \%$. The cumulative amount of endostar released from the carriers in phosphate-buffered saline over 21 days was $23.79 \%, 20.45 \%, 15.13 \%$, and $10.41 \%$, respectively. Moreover, the terminal elimination halflife of endostar in the rabbit was $26.91 \pm 7.93$ hours and $9.32 \pm 5.53$ hours in the PEG-PLGA group and the PLGA nanoparticle group. Peak endostar concentration was reached at day 7 in the group treated with subcutaneous injection of PEG-PLGA microspheres and at day 14 in the group receiving subcutaneous injection of PLGA microspheres. Endostar was detectable in vivo in both groups after injection of the particulate carriers.

Conclusion: PEG-PLGA nanoparticles might be better than other nanoparticulate carriers for encapsulation and distribution of endostar.

Keywords: poly(DL-lactide-co-glycolide), nanoparticle, microsphere, endostar, peptide delivery

\section{Introduction}

Endostar, a novel recombinant human endostatin, has been approved by the Chinese State Food And Drug Administration for the treatment of nonsmall cell lung cancer. Endostar has a broad spectrum of activity against solid tumors, by inhibiting endothelial cell proliferation, migration, and vessel formation. ${ }^{1-4}$ Despite its apparent therapeutic value, the biological half-life of endostar, like most other protein drugs, is short because of its rapid metabolism. Multiple injections of endostar at a high dose $\left(7.5 \mathrm{mg} / \mathrm{m}^{2} /\right.$ day in the first 2 weeks of a 3 -week treatment cycle), in order to maintain therapeutic levels, are still associated with fluctuating plasma endostar concentrations. ${ }^{5,6}$ It is expected that a long-acting formulation of endostar would provide stable antitumor activity and result in fewer injection-related complications. ${ }^{7}$ 
Several attempts have been made to generate long-acting forms of endostatin, including alteration of the molecule to increase its stability, direct poly(ethylene glycol) (PEG) modification of the peptide, and other approaches. ${ }^{8}$ Mutagenesis and modification of the molecule is difficult because the bioactivity and efficacy of the peptide must be maintained, and integrity of multiple active sites and conformation must be preserved. Recently, endostar-loaded microspheres and PEG-modified poly(DL-lactide-co-glycolide) (PEG-PLGA) nanoparticles were prepared, with higher acid resistance and isoelectric point, and more stability of endostar resulting from an additional nine amino acid sequence at the $\mathrm{N}$ terminus. ${ }^{9-12}$ In addition, particulate carriers represent new approaches to improving the pharmacodynamic properties of therapeutic peptides, in that they can encapsulate and deliver drugs, and hydrolyze in an aqueous environment (hydrolytic degradation or biodegradation) in a stable manner. ${ }^{13}$ Unlike other approaches, such as mutagenesis, direct PEGylation, and fusion to carrier proteins, particulate carriers have not been shown to change the amino acid sequence of any of the proteins involved.

The purpose of this study was to identify a suitable carrier for encapsulation and delivery of endostar. Because the physicochemical and biological properties of peptide drugs are different from those of other agents, including molecular size, biological half-life, conformation stability, physicochemical stability, and bioavailability, the design and preparation of these carriers is different. Liposomes, microspheres, and nanoparticles are mostly used for peptide drug delivery. ${ }^{11}$ The structure, charge, solubility, and other properties, including the liposomes used for the different peptides vary and the formulation methods and materials are different. ${ }^{14}$ Moreover, liposomes are used more often for encapsulation of lipid-soluble medicines because of their fat solubility. Therefore, we prepared endostar-loaded nanoparticles and microspheres, and assessed their ability to prolong the circulation time of endostar.

\section{Materials and methods Materials}

Endostar $5 \mathrm{mg} / \mathrm{mL}$ was obtained from Shandong Simcere Medgenn BioPharmaceutical Co Ltd (Yantai, China). PLGA (lactide:glycolide ratio 50:50, molecular weight $45 \mathrm{kDa}$ ) and PEG-PLGA (PEG molecular weight $2 \mathrm{kDa}$ and PLGA molecular weight $45 \mathrm{kDa}$ ) were purchased from Shandong Institute of Medical Instruments (Shandong, China). ${ }^{12}$ PEG and PLGA are nontoxic, nonimmunogenic, and biodegradable, with the potential for controlled release of injected drugs and site-specific drug delivery. ${ }^{15,16}$ Polyvinyl alcohol (PVA, molecular weight $13-23 \mathrm{kDa}$ ) and the primary antibodies for endostatin were purchased from Sigma-Aldrich (St Louis, MO) and Santa Cruz Biotechnology (Santa Cruz, CA), respectively. All other chemicals used were of analytical grade. The apparatus, including transmission electron microscopy (Philips, the Netherlands), microscopy (Jiangnan Optical-Electrical Co Ltd, Nanjing, China), photon correlation spectroscopy (Malvern, Worcestershire, UK), ultraviolet spectrophotometry (Spectrum China Ltd, Shanghai, China), low-temperature ultracentrifugation (Hitachi, Japan), and enzyme-linked immunosorbent assay (Bio-Tek, Winooski, VT) were used. New Zealand rabbits $(2-3 \mathrm{~kg})$ were purchased from the Animal Center of Shandong University, China. All experiments performed with animals were approved by the ethics committee of Shandong University.

\section{Endostar-loaded PLGA and PEG-PLGA nanoparticles and microspheres}

The PLGA and PEG-PLGA nanoparticles were prepared by a double emulsion (mixing solvent) as described in previous studies. ${ }^{12,17,18}$ This preparation technique was adapted to obtain nanoparticles and microspheres. Briefly, the first emulsion $(\mathrm{o} / \mathrm{w})$ was formed between a dichloromethane solution of PLGA or PEG-PLGA $5 \mathrm{mg} / \mathrm{mL}$ and endostar $(0.05 \mathrm{~mL}$, $5 \mathrm{mg} / \mathrm{mL}$ ) by high-speed shearing ( $2800 \mathrm{rpm}$ for 60 seconds). Subsequently, a volume of $2 \mathrm{~mL}$ of $0.1 \%$ (w/v) PVA was added into this primary w/o emulsion to obtain the double emulsion (w/o/w) by high-speed shearing $(25,000 \mathrm{rpm}$ for 60 seconds). The solvent was evaporated in $10 \mathrm{~mL}$ of aqueous solution $(0.1 \%$, PVA) by gentle magnetic stirring (1000 rpm) at room temperature. The nanoparticles were recovered by centrifugation (40,000 rpm for 40 minutes), and washed three times in phosphate-buffered saline.

The PLGA and PEG-PLGA microspheres were also prepared by a double emulsion method, which was slightly different from that used to create the nanoparticles. ${ }^{11}$ The primary w/o emulsion was emulsified at $400 \mathrm{rpm}$ for 30 seconds, added to $50 \mathrm{~mL}$ of aqueous solution containing $0.1 \%$ PVA, and stirred at $900 \mathrm{rpm}$ for 4 hours at room temperature to evaporate the organic solvents. The microspheres were collected by centrifugation at $5000 \mathrm{rpm}$ for 3 minutes, washed three times, and freeze-dried.

\section{Physicochemical characterization}

The entrapment efficiency of particulate carriers was assayed using a direct method. After dissolving the lyophilized nanospheres or microspheres in $0.05 \mathrm{~N} \mathrm{NaOH}$ and $1 \%$ sodium dodecyl sulfate, the endostar content was estimated using the micro bicinchoninic acid protein assay, which was validated using purified endostar. ${ }^{12,19}$ The lower limit of detection was 
$0.005 \mu \mathrm{g} / \mathrm{mL}$. No interference with PEG-PLGA, PLGA, or the stabilizers was observed. The drug-loading capacity and entrapment efficiency were calculated as follows:

$$
\begin{aligned}
\text { Endostar loading capacity }(\%)= & \mathrm{M}_{\text {endostar }} / \mathrm{M}_{\text {endostar-loaded particulate carriers }} \\
& \times 100 \\
\mathrm{EE}(\%)= & \mathrm{M}_{\text {endostar }} / \mathrm{M}_{\text {endostar devoted }} \times 100
\end{aligned}
$$

where EE is entrapment efficiency, $M_{\text {endostar }}$ is the amount of drug in the nanoparticles or microspheres $\left(\mathrm{M}_{\text {endostar }}=\right.$ $\mathrm{C} \times \mathrm{V}, \mathrm{C}$ is concentration in the supernatant, $\mathrm{V}$ is volume), $\mathrm{M}_{\text {endostar-loaded particulate carriers }}$ is the amount of nanoparticles or microspheres containing endostar, and $\mathrm{M}_{\text {endostar devoted }}$ is the initial amount of endostar.

Morphology of the nanoparticles was observed by transmission electron microscopy, and particle size distribution (mean diameter and polydispersity index) was determined by photon correlation spectroscopy. The size of the microspheres was determined using a microscope whereby 200 particles were counted to calculate the average diameter and percentage distribution of the microspheres. ${ }^{12}$

The presence of residual PVA on the surface of the particles was determined by both direct and indirect methods. ${ }^{20}$ The nanoparticles or microspheres were digested in $0.05 \mathrm{~N} \mathrm{NaOH}$ and $1 \%$ sodium dodecyl sulfate. The solution obtained was then neutralized and analyzed for PVA content using the colorimetric method. ${ }^{21}$ Residual PVA was also calculated according to the difference between the total amount used and the amount present in the supernatant during the washing steps. The average of the results obtained by the two methods was used.

\section{Release studies in phosphate-buffered saline}

The nanoparticles or microspheres were also evaluated for their in vitro release characteristics using the horizontal shaker method. ${ }^{22}$ Endostar-loaded nanoparticles or microspheres were suspended in phosphate-buffered saline ( $\mathrm{pH} 7.4,0.01 \%$ sodium azide, $0.02 \%$ Tween 80 ) in an Eppendorf dialysis tube, and stirred at $37^{\circ} \mathrm{C} \pm 0.5^{\circ} \mathrm{C}$ and $100 \mathrm{rpm}$. At appropriate intervals, we centrifuged the suspension, collected the supernatant, and added fresh phosphate-buffered saline dialysis buffer to the tube. The amount of endostar in the supernatants was determined using the micro bicinchoninic acid protein assay. Subsequently, the endostar release profiles were expressed in terms of cumulative protein release versus time.

\section{In vivo release studies}

The in vivo evaluation was performed using 30 male New Zealand rabbits, which were randomly divided into six groups and housed individually in a room at constant ambient temperature and humidity. After an overnight fast, a bolus of endostar, endostar-loaded PLGA, or PEG-PLGA nanoparticles containing endostar $90 \mathrm{mg} / \mathrm{m}^{2}$ was administered through the aural vein. In the other three groups, the same amount of endostar or endostar-loaded PLGA and PEG-PLGA microspheres was given subcutaneously at the back of the neck. Blood samples were collected from the aural vein at a series of time intervals. The endostar concentration in plasma was determined using an enzyme-linked immunosorbent assay, which was validated using purified recombinant human endostatin with a detection limit of $0.002-0.5 \mu \mathrm{g} / \mathrm{mL}$.

\section{Amount of endostar in different tissues in vivo}

The amount of endostar in the different tissues was detected by Western blot assay using a polyclonal antiendostatin antibody for investigating clearance efficacy in the rabbit. ${ }^{12,23}$ Fifteen New Zealand rabbits, divided into three groups, were given the same dose of endostar, endostar-loaded PEGPLGA, or PLGA nanoparticles at $180 \mathrm{mg} / \mathrm{m}^{2}$. Tissue samples of liver, spleen, and lung were collected, weighed, and homogenated when the rabbits were sacrificed. The amount of endostar was determined by Western blot assay.

\section{Statistical analysis}

All experiments were performed in triplicate, and data are shown as means \pm standard deviation. The Student's $t$-test was used to compare the treatment groups. $P<0.05$ was considered to be statistically significant in all cases. The pharmacokinetics of endostar distribution were calculated using the DAS 2.0 program. ${ }^{23}$

\section{Results Physicochemical characterization}

The endostar-loaded nanoparticles and microspheres were found to have a spherical core shell structure with a relatively smooth surface. Although the size of the PEG-PLGA nanoparticles was similar to that of the PLGA nanoparticles $(147.32 \pm 31.93 \mathrm{~nm}$ vs $127.31 \pm 26.18 \mathrm{~nm}$, respectively, $P>0.05)$, the loading capacity and encapsulation efficiency was greater than for the PLGA nanoparticles $(8.03 \% \pm$ $1.21 \%$ vs $3.27 \% \pm 1.36 \%, P<0.05)$. The size of the PEGPLGA nanoparticles was smaller than that of the PLGA microspheres $(147.32 \pm 31.93 \mathrm{~nm}$ vs $27.45 \pm 4.26 \mu \mathrm{m}$, $P<0.01$ ), but there was no significant difference between the PEG-PLGA nanoparticles and PLGA microspheres with regard to their loading capacity and encapsulation efficiency 
Table I Physicochemical characteristics of the nanoparticles and microspheres

\begin{tabular}{lccllll}
\hline Formulation & Particle size & Loading (\%) & EE (\%) & PVA content (\%) & Zeta potential (mV) & PDI \\
\hline PEG-PLGA nanoparticles $(\mathrm{nm})$ & $147.32 \pm 31.93$ & $8.03 \pm 1.21$ & $75.97 \pm 8.67$ & $0.6 \pm 1.1$ & $-16.2 \pm 0.3$ & $0.39 \pm 0.15$ \\
PLGA nanoparticles $(\mathrm{nm})$ & $127.31 \pm 26.18$ & $3.27 \pm 1.36^{*}$ & $65.45 \pm 9.62^{*}$ & $6.3 \pm 2.5^{*}$ & $-33.3 \pm 0.2$ & $0.31 \pm 0.18$ \\
PEG-PLGA microspheres $(\mu \mathrm{m})$ & $45.79 \pm 6.87^{*}$ & $15.32 \pm 1.21^{*}$ & $91.84 \pm 3.29^{*}$ & $0.3 \pm 0.6$ & $-25.1 \pm 0.5$ & $0.46 \pm 0.19$ \\
PLGA microspheres $(\mu \mathrm{m})$ & $27.45 \pm 4.26^{*}$ & $9.21 \pm 1.73$ & $82.74 \pm 5.67$ & $3.2 \pm 1.9 *$ & $-57.9 \pm 0.6$ & $0.73 \pm 0.22$
\end{tabular}

Note: *Significantly different from PEG-PLGA nanoparticles $(P<0.05)$.

Abbreviations: EE, entrapment efficiency; PLGA, poly(DL-lactide-co-glycolide); PEG-PLGA, poly(ethylene glycol) modified poly(DL-lactideco-glycolide); PVA, polyvinyl alcohol; PDI, polydispersity index.

$(8.03 \% \pm 1.21 \%$ vs $9.21 \% \pm 1.73 \%, P>0.05)$. The residual PVA content of the PEG-PLGA nanoparticles was negligible compared with that on the PLGA nanoparticle surface $(0.6 \% \pm 1.1 \%$ vs $6.3 \% \pm 2.5 \%, P<0.05)$. The amount of PVA on the PEG-PLGA microspheres was also less than that of the PLGA microspheres $(0.3 \% \pm 0.6 \%$ vs $3.2 \% \pm 1.9 \%$, $P<0.05)$. The particle size, loading capacity, encapsulation efficiency, PVA content, zeta potential, and polydispersity index of the particulate carriers are shown in Table 1.

\section{Release studies in phosphate-buffered saline}

Endostar was released more rapidly from the nanoparticles than the microspheres, and the release speed from the PEG-PLGA nanoparticles was higher than that from the PLGA nanoparticles from the third release day onwards. The PEG-PLGA nanoparticles released more encapsulated endostar over the same time period. Endostar release from the endostar-loaded nanoparticles and microspheres ( $\mathrm{pH} 7.04)$ in vitro was biphasic, with an initial abrupt release, followed by more sustained release (Figure 1). In the initial burst release, a significant amount of endostar was released within 24 hours (9.27\% for the PEG-PLGA nanoparticles, $10.56 \%$ for the PLGA nanoparticles, 5.02\% for the PEG-PLGA microspheres, and $6.67 \%$ for the PLGA microspheres). Subsequently, the endostar displayed a sustained release profile. Cumulative endostar release over 21 days was $24.15 \%$ for the PEG-PLGA nanoparticles, $20.31 \%$ for the PLGA nanoparticles, $14.35 \%$ for the PEG-PLGA microspheres, and $10.32 \%$ for the PLGA microspheres.

\section{Release studies in vivo}

Endostar concentrations in rat plasma in the PEG-PLGA nanoparticle-treated group could be maintained at high levels. Although the concentration of endostar in blood 10 minutes after intravenous administration in the endostar group was about four times that in the PEG-PLGA nanoparticle group, it was quickly removed from the circulation in the endostar only group, with a terminal elimination half-life of $2.72 \pm 1.43$ hours. While the endostar-loaded PEG-PLGA and PLGA nanoparticles exhibited markedly delayed blood clearance, the elimination half-life of endostar was $26.93 \pm 7.93$ hours and $9.35 \pm$ 5.51 hours, respectively $(P<0.05)$. The areas under the curve for endostar in the PEG-PLGA, PLGA nanoparticle, and endostar groups were $69821.95 \pm 6219.84 \mu \mathrm{g} / \mathrm{L} *$ hour, $15238.77 \pm$ $5916.18 \mu \mathrm{g} / \mathrm{L} *$ hour, and $23396.31 \pm 7951.65 \mu \mathrm{g} / \mathrm{L}^{*}$ hour, respectively. Mean plasma concentrations for the endostar and endostar-loaded nanoparticles in rabbits are shown in Figure 2. The endostar-loaded microspheres also showed a sustained release profile whereby the peak concentration was reached on day 7 for the PEG-PLGA microspheres and on day 14 for the PLGA microspheres, and was maintained until day 27 (Figure 3). The peak concentration of endostar in the PEG-PLGA microsphere group was higher than that in the PLGA microsphere group $(0.3974 \pm 0.0316 \mu \mathrm{g} / \mathrm{mL}$ vs $0.3015 \pm 0.0276 \mu \mathrm{g} / \mathrm{mL}, P<0.05)$.

\section{Amount of endostar in different tissues in vivo}

The amount of endostar in different tissues in the PEGPLGA nanoparticle group was greater than in the PLGA or

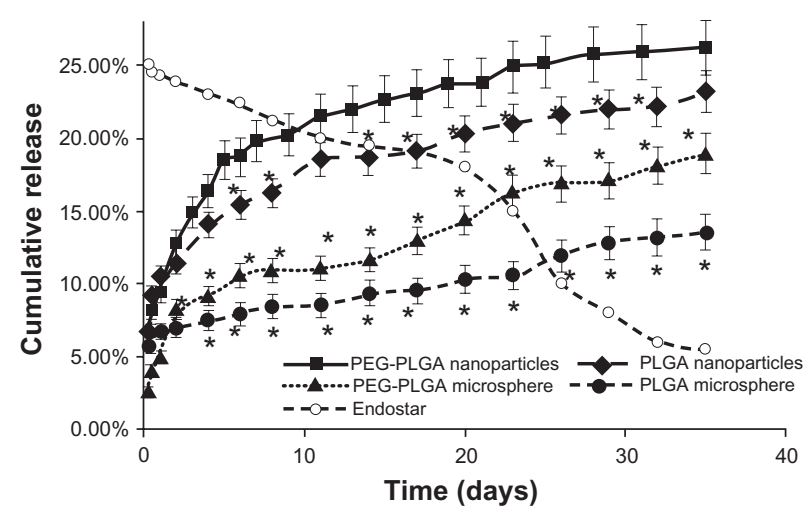

Figure I Release profiles of endostar from PLGA nanoparticles, PEG-PLGA nanoparticles, and PLGA and PEG-PLGA microspheres in phosphate-buffered saline. The error bars represent means \pm standard deviation. The dissolution of endostar with a $25 \%$ concentration is also described.

Note: *Significantly different from PEG-PLGA nanoparticles $(P<0.05)$. 


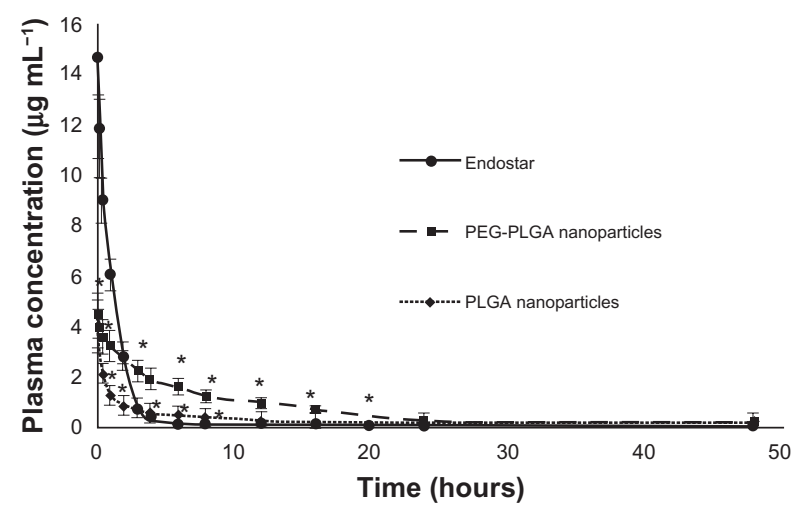

Figure 2 Mean plasma concentration of endostar following a single intravenous administration of endostar, endostar-loaded PEG-PLGA nanoparticles or PLGA nanoparticles at $90 \mathrm{mg} / \mathrm{m}^{2}$ in rabbits.

Note: *Significantly different from PEG-PLGA nanoparticles $(P<0.05)$.

Abbreviations: PLGA, poly(DL-lactide-co-glycolide); PEG-PLGA, poly(ethylene glycol) modified poly(DL-lactide-co-glycolide)

microsphere groups. The amount of endostar was assayed by Western blot assay. ${ }^{12}$ Endostar was detectable only in the endostar-containing PEG-PLGA group and PLGA nanoparticle group 5 hours after intravenous nanoparticle injection and was not detectable after subcutaneous microsphere injection (Figure 4). The relative tissue content of endostar (endostar: actin, \%) in the endostar-loaded PEG-PLGA nanoparticle group was $200.5 \pm 12.5$ in the liver, $216.3 \pm 15.2$ in the spleen, and $176.7 \pm 8.6$ in the lung, and was $109 \pm 6.6$, $91 \pm 5.7$, and $67 \pm 4.4$, respectively, in the endostar-loaded PLGA nanoparticle group. Endostar was not detectable in the endostar or microsphere groups, even at day 7 after subcutaneous injection.

\section{Discussion}

Both nanoparticles and microspheres can be developed using a double emulsion method, with similar release

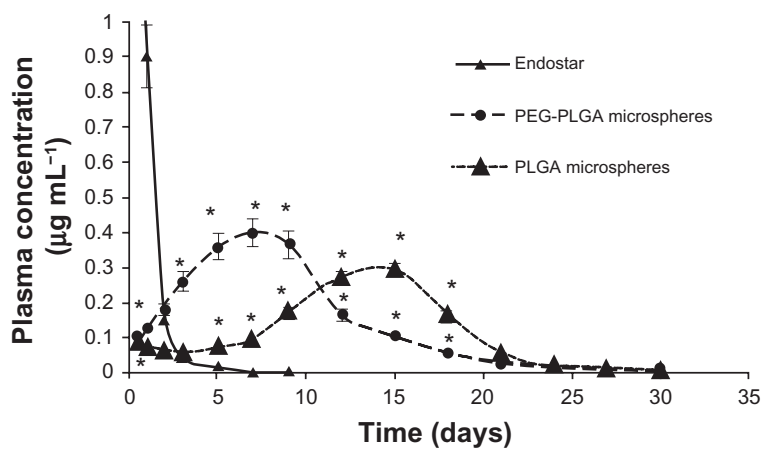

Figure 3 Mean plasma concentration of endostar following a single subcutaneous injection of endostar, endostar-loaded PEG-PLGA microspheres, or PLGA microspheres at $90 \mathrm{mg} / \mathrm{m}^{2}$ in rabbits.

Note: *Significantly different from PEG-PLGA nanoparticles $(P<0.05)$.

Abbreviations: PLGA, poly(DL-lactide-co-glycolide); PEG-PLGA, poly(ethylene glycol) modified poly(DL-lactide-co-glycolide).

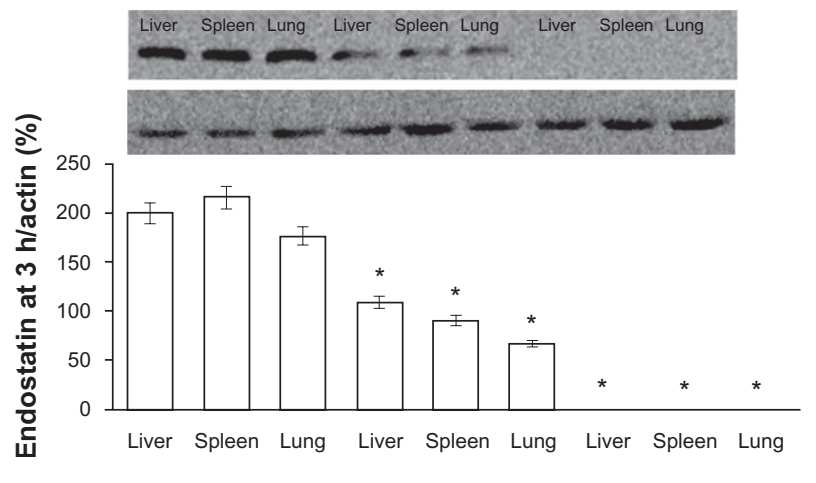

Figure 4 The amount of endostar in tissues following a single injection of endostarloaded PEG-PLGA, PLGA nanoparticles, and endostar. The left three bands were from the endostar-loaded PEG-PLGA nanoparticle group, the middle three ones from the PLGA nanoparticle group, and the right ones from the endostar group. Endostar was not detectable in the endostar, endostar-loaded PLGA, or PEG-PLGA microsphere groups.

Note: *Significantly different from PEG-PLGA nanoparticles $(P<0.05)$.

Abbreviations: PLGA, poly(DL-lactide-co-glycolide); PEG-PLGA, poly(ethylene glycol) modified poly(DL-lactide-co-glycolide).

characteristics. ${ }^{11,12,24}$ However, there are still some differences between these two carriers. Table 1 shows higher encapsulation efficiencies of endostar in microspheres and PEG-PLGA particulate carriers, and less residual PVA content in PEG-PLGA particulate carriers. This may be attributed to the larger size and smaller surface area that enables the microspheres to encapsulate more drug and adsorb less PVA than the nanoparticles. ${ }^{25}$ The higher encapsulation rate for the endostar-loaded PEG-PLGA nanoparticles might result from the hydrophilic moiety on PEG. This hydrophilic moiety on the surface can more easily bind and encapsulate soluble endostar into particulate carriers. Less residual PVA on the surface of the PEG-PLGA nanoparticles or microspheres confirmed that PEG-PLGA was more hydrophilic than PLGA and hinders surface adsorption of PVA. ${ }^{20}$ Thus, the encapsulation efficiency of PEG-PLGA nanoparticles was high.

The release speeds of the nanoparticles and microspheres were also different. PEG-PLGA or PLGA nanoparticulate carriers would hydrolyze in an aqueous environment (hydrolytic degradation or biodegradation), and release the encapsulated drug. ${ }^{13}$ The biodegradation rate of the particulate carriers was dependent on the hydrophilic/lipophilic ratio of the polymer and surface area. The more hydrophilic and the larger the surface area of the polymer, the more rapid its degradation..$^{26}$ Thus, the presence of PEG had a positive effect on degradation of the nanoparticles and microspheres. As can be seen in Figure 2, higher total amounts of endostar were released from the PEG-PLGA nanoparticles and microspheres (Figure 2). The higher release effect observed for the nanoparticles as compared with the microspheres might be attributed to their larger surface area. Thus, different 
particulate carriers are chosen to control the release of drugs, modify their pharmacokinetics, enhance their anticancer effect, and decrease their toxicity. ${ }^{27-29}$ The PEG-PLGA nanoparticles encapsulated more endostar and released endostar more rapidly than the other three carriers.

Microsphere carriers were not suitable for loading endostar. Most drug-loaded microspheres are too large in diameter to be administered intravenously, because largesized particulate carriers embolize easily in the blood vessels, triggering an opsonization effect in the immune system and they also have difficulty crossing endothelial barriers in various hosts. ${ }^{30}$ Moreover, the effect of some medicines injected subcutaneously shows significant variation at different injection sites in different individuals, and this is attributed to variation in body response. ${ }^{31-33}$ Because of the presence of proteolytic enzymes, cellular infiltrates, various cytokines, and $\mathrm{pH}$ gradients, subcutaneous conditions are complex in vivo. The release profile of endostar from the microspheres confirmed that the concentration levels of endostar were fluctuant and lower (Figure 3), and not detectable in tissues (data not shown).

However, the intravenously administered nanoparticles accumulated more easily in tissues, and the plasma concentration of endostar was higher. Five hours after intravenous or subcutaneous injection, endostar was only detectable in the endostar-containing PEG-PLGA and PLGA nanoparticle groups, and not detectable in the endostar only or microsphere groups. The relative content of endostar in the endostar-loaded PEG-PLGA nanoparticle group was more than that in the endostar-loaded PLGA nanoparticle group. Furthermore, the elimination half-life of endostar in the PEG-PLGA nanoparticle group was longer than that in the PLGA nanoparticle or endostar groups $(P<0.05)$. These observations might result from PEG having a hydrophilic moiety which can prevent interactions with other cells and proteins, ${ }^{15,16}$ being cleared from the systemic circulation, and being transported in the lymphatic system. Studies have demonstrated that nanoparticles with a PEG layer $100 \mathrm{~nm}$ thick are not easily engulfed by phagocytes. ${ }^{34,35}$ Moreover, PEG-PLGA nanoparticles might also accumulate easily in tumor tissue. As a result of the enhanced permeability of tumor endothelium and lack of lymphatic drainage in tumor cells, there is increased extravasation and accumulation of drug in tumor vasculature and tumor tissue. In addition, endothelial cells in tumors are distinct from those in normal tissues, possessing wide fenestrations, ranging from $200 \mathrm{~nm}$ to $1.2 \mathrm{~mm}$ in size. The vascular pore size of the LS174T tumor, a human colon adenocarcinoma, may be as large as $400 \mathrm{~nm} .{ }^{36}$ This large pore size allows passage of nanoparticles into the extravascular spaces, which is known as the "enhanced permeation and retention" effect. Thus, the PEG-PLGA nanoparticles accumulate more easily in tumor tissue.

\section{Conclusion}

PEG-PLGA nanoparticles can maintain good concentrations of endostar in plasma and tissues, and show more promising characteristics than PLGA nanoparticles and microspheres. Although more investigations need to be done, PEG-PLGA nanoparticles seem to represent a suitable endostar carrier system. PEG-PLGA nanoparticles might also become novel carriers for other peptide medicines, providing new opportunities to control delivery of peptide drugs.

\section{Acknowledgment}

This work was supported by the National High Technology Research and Development Program 863 Fund of China (No. 2007AA021802).

\section{Disclosure}

The authors report no conflicts of interest in this work.

\section{References}

1. Zhou ZT, Zhou FX, Wei Q, et al. Phase II study of cisplatin/etoposide and endostar for extensive-stage small-cell lung cancer. Cancer Chemother Pharmacol. 2011. [Epub ahead of print].

2. Ling Y, Yang Y, Lu N, et al. Endostar, a novel recombinant human endostatin, exerts antiangiogenic effect via blocking VEGF-induced tyrosine phosphorylation of KDR/Flk-1 of endothelial cells. Biochem Biophys Res Commun. 2007;361:79-84.

3. Zhuo W, Luo C, Wang X, Song X, Fu Y, Luo Y. Endostatin inhibits tumour lymphangiogenesis and lymphatic metastasis via cell surface nucleolin on lymphangiogenic endothelial cells. J Pathol. 2010;222:249-260.

4. Han B, Xiu Q, Wang H, et al. A multicenter, randomized, doubleblind, placebo-controlled study to evaluate the efficacy of paclitaxelcarboplatin alone or with endostar for advanced non-small cell lung cancer. J Thorac Oncol. 2011;6:1104-1109.

5. Kisker O, Becker CM, Prox D, et al. Continuous administration of endostatin by intraperitoneally implanted osmotic pump improves the efficacy and potency of therapy in a mouse xenograft tumor model. Cancer Res. 2001;61:7669-7674.

6. Xu F, Ma Q, Sha H. Optimizing drug delivery for enhancing therapeutic efficacy of recombinant human endostatin in cancer treatment. Crit Rev Ther Drug Carrier Syst. 2007;24:445-492.

7. Kuroiwa M, Takeuchi T, Lee JH, et al. Continuous versus intermittent administration of human endostatin in xenografted human neuroblastoma. J Pediatr Surg. 2003;38:1499-1505.

8. Tong Y, Zhong K, Tian H, et al. Characterization of a monoPEG20000Endostar. Int J Biol Macromol. 2010;46:331-336.

9. Song HF, Liu XW, Zhang HN, et al. Pharmacokinetics of His-tag recombinant human endostatin in Rhesus monkeys. Acta Pharmacol Sin. 2005;26:124-128.

10. Jiang LP, Zou C, Yuan X, Luo W, Wen Y, Chen Y. N-terminal modification increases the stability of the recombinant human endostatin in vitro. Biotechnol Appl Biochem. 2009;54:113-120. 
11. Meng B, Li L, Hua S, et al. Effect of medium-chain triglycerides on the release behavior of Endostar encapsulated PLGA microspheres. Int $J$ Pharm. 2010;397:136-143.

12. Hu S, Zhang Y. Endostar-loaded PEG-PLGA nanoparticles: in vitro and in vivo evaluation. Int J Nanomedicine. 2010;5:1039-1048.

13. Muthu M. Nanoparticles based on PLGA and its co-polymer: an overview. Asian Journal of Pharmaceutics. 2009;3:266-273.

14. Tan ML, Choong PF, Dass CR. Recent developments in liposomes, microparticles and nanoparticles for protein and peptide drug delivery. Peptides. 2010;31:184-193.

15. Wang M, Thanou M. Targeting nanoparticles to cancer. Pharmacol Res. 2010;62:90-99.

16. Li SD, Huang L. Nanoparticles evading the reticuloendothelial system: role of the supported bilayer. Biochim Biophys Acta. 2009; 1788:2259-2266.

17. Cohen S, Yoshioka T, Lucarelli M, Hwang LH, Langer R. Controlled delivery systems for proteins based on poly(lactic/glycolic acid) microspheres. Pharm Res. 1991;8:713-720.

18. Roy A, Singh MS, Upadhyay PK, Bhaskar S. Combined chemoimmunotherapy as a prospective strategy to combat cancer: a nanoparticle based approach. Mol Pharm. 2010. [Epub ahead of print].

19. Mukherjee B, Santra K, Pattnaik G, Ghosh S. Preparation, characterization and in-vitro evaluation of sustained release protein-loaded nanoparticles based on biodegradable polymers. Int J Nanomedicine. 2008;3:487-496

20. Gref R, Quellec P, Sanchez A, Calvo P, Dellacherie E, Alonso MJ. Development and characterization of CyA-loaded poly(lactic acid)poly(ethylene glycol)PEG micro- and nanoparticles. Comparison with conventional PLA particulate carriers. Eur J Pharm Biopharm. 2001;51:111-118.

21. Zwick MM. The blue complexes of iodine with poly(vinyl alcohol) and amylose. J Polym Sci A1. 1966;4:1642-1644.

22. Wu J, Wu L, Xu X, et al. Microspheres made by w/o/o emulsion method with reduced initial burst for long-term delivery of endostar, a novel recombinant human endostatin. J Pharm Sci. 2009;98:2051-2058.

23. Thomas JP, Arzoomanian RZ, Alberti D, et al. Phase I pharmacokinetic and pharmacodynamic study of recombinant human endostatin in patients with advanced solid tumors. J Clin Oncol. 2003;21:223-231.
24. Wu J, Ding D, Ren G, Xu X, Yin X, Hu Y. Sustained delivery of endostatin improves the efficacy of therapy in Lewis lung cancer model. J Control Release. 2009;134:91-97.

25. Panyam J, Dali MM, Sahoo SK, et al. Polymer degradation and in vitro release of a model protein from poly(D,L-lactide-co-glycolide) nano- and microparticles. J Control Release. 2003;92:173-187.

26. Jain RA. The manufacturing techniques of various drug loaded biodegradable poly(lactide-co-glycolide) (PLGA) devices. Biomaterials. 2000;21:2475-2490.

27. Peer D, Karp JM, Hong S, Farokhzad OC, Margalit R, Langer R. Nanocarriers as an emerging platform for cancer therapy. Nat Nanotechnol. 2007;2:751-760.

28. Wang MD, Shin DM, Simons JW, Nie S. Nanotechnology for targeted cancer therapy. Expert Rev Anticancer Ther. 2007;7:833-837.

29. Petros RA, DeSimone JM. Strategies in the design of nanoparticles for therapeutic applications. Nat Rev Drug Discov. 2010;9:615-627.

30. Moghimi SM, Szebeni J. Stealth liposomes and long circulating nanoparticles: critical issues in pharmacokinetics, opsonization and protein-binding properties. Prog Lipid Res. 2003;42:463-478.

31. Tracy MA, Ward KL, Firouzabadian L, et al. Factors affecting the degradation rate of poly(lactide-co-glycolide) microspheres in vivo and in vitro. Biomaterials. 1999;20:1057-1062.

32. Ingvast-Larsson $\mathrm{C}$, Holgersson $\mathrm{A}$, Bondesson $\mathrm{U}$, Lagerstedt AS, Olsson K. Clinical pharmacology of methadone in dogs. Vet Anaesth Analg. 2010;37:48-56.

33. Kapitza C, Zdravkovic M, Zijlstra E, Segel S, Heise T, Flint A. Effect of three different injection sites on the pharmacokinetics of the once-daily human GLP-1 analogue liraglutide. J Clin Pharmacol. 2011;51:951-955.

34. Jeong YI, Kang MK, Sun HS, et al. All-trans-retinoic acid release from core-shell type nanoparticles of poly(epsilon-caprolactone)/poly(ethylene glycol) diblock copolymer. Int J Pharm. 2004;273:95-107.

35. Zhang X, Pan SR, Hu HM, et al. Poly(ethylene glycol)-blockpolyethylenimine copolymers as carriers for gene delivery: effects of PEG molecular weight and PEGylation degree. J Biomed Mater Res A. 2008;84:795-804.

36. Hobbs SK, Monsky WL, Yuan F, et al. Regulation of transport pathways in tumor vessels: role of tumor type and microenvironment. Proc Natl Acad Sci U S A. 1998;95:4607-4612.
International Journal of Nanomedicine

\section{Publish your work in this journal}

The International Journal of Nanomedicine is an international, peerreviewed journal focusing on the application of nanotechnology in diagnostics, therapeutics, and drug delivery systems throughout the biomedical field. This journal is indexed on PubMed Central,

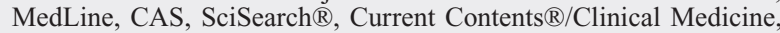

\section{Dovepress}

Journal Citation Reports/Science Edition, EMBase, Scopus and the Elsevier Bibliographic databases. The manuscript management system is completely online and includes a very quick and fair peer-review system, which is all easy to use. Visit http://www.dovepress.com/ testimonials.php to read real quotes from published authors. 